4. A. Garsia, S. Orey and E. Rodemich, Asymptotic behavior of successive coeffcients of some power series, Illinois J. Math. 6 (1962), 620-629.

5. S. Karlin and J. McGregor, Random walks, Illinois J. Math. 3 (1959), 66-81.

6. D. G. Kendall, Unitary dilations of Markov transition operators, and the corresponding integral representation for transition-probability matrices, Harold Cramer Volume (U. Grenander, Ed.), Almquist and Wiksell, Stockholm, 1960; pp. 139-161.

7. S. Orey, Strong ratio limit property, Bull. Amer. Math. Soc. 67 (1961), 571-574.

8. D. Vere-Jones, Geometric ergodicity in denumerable Markov chains, Quart. J. Math. Oxford Ser. (2) 13 (1962), 7-28.

UnIVERSITY OF MinNESOTA

\title{
A REPRESENTATION THEOREM FOR CONTINUOUS FUNCTIONS OF SEVERAL VARIABLES
}

\author{
DAVID A. SPRECHER ${ }^{1}$
}

Let $E^{n}$ denote the $n$-dimensional unit cube in Euclidean space; designate the closed unit interval, $[0,1]$, by $E$. We prove in this note the following

THEOREM. For any natural number $n, n \geqq 2$, there exist real, monotonic increasing functions, $h^{p}(x), 1 \leqq p \leqq n$, dependent on $n$, and having the following properties:

(i) The function

$$
\sum_{1 \leq p \leq n} h^{p}\left(x_{p}\right)
$$

separates all points of $E^{n}$ :

$$
\sum_{1 \leq p \leq n} h^{p}\left(x_{p}\right) \neq \sum_{1 \leq p \leq n} h^{p}\left(y_{p}\right)
$$

unless $x_{p}=y_{p}$ for all admitted values of $p$.

(ii) Every continuous function of $n$ variables, $f\left(x_{1}, \cdots, x_{n}\right)$, with domain $E^{n}$, can be represented in the form

$$
f\left(x_{1}, \cdots, x_{n}\right)=g\left[\sum_{1 \leq p \leq n} h^{p}\left(x_{p}\right)\right] .
$$

Clearly, the function $g$ will, in general, be discontinuous.

Received by the editors September 16, 1963.

1 This note is part of the author's Ph.D. thesis (University of Maryland, 1963, directed by A. Douglis); its research was supported by the United States Air Force through the AFOSR under Contract No. AF 49(638)-590. 
As shown by V. I. Arnol'd [1], even a simple function such as $f(x, y)=x y$ cannot be represented in the form (1) if the functions $g$ and $h^{p}$ are required to be continuous.

Proof. We represent the real numbers in the interval $E$ to the base $n$ :

$$
x=\sum_{1 \leq \nu \leq \infty} s_{\nu} \cdot n^{-r},
$$

where $s_{\nu}$ is an index with domain $0 \leqq s_{\nu} \leqq n-1$ for each $\nu$. To have a one-to-one correspondence between the real numbers, $x \in E$, and the infinite series (2), we normalize (2) by requiring that for no $N>1$ is $s_{\nu}=n-1$ for all $\nu \geqq N$, except when $s_{\nu}=n-1$ for all $\nu$.

To prove (i), we set for each admitted $p$

$$
x_{p}=\sum_{1 \leq \nu \leq \infty} s_{p \nu} \cdot n^{-r}
$$

where $0 \leqq s_{p \nu} \leqq n-1$, the $s_{p \nu}$ being subject to the normalizing restriction just described, and define the functions

$$
h^{p}\left(x_{p}\right)=\sum_{1 \leq \nu \leq \infty} s_{p \nu} \cdot n^{-n v-p+1} .
$$

Clearly, (4) is a representation of real numbers in $E$ to the base $n^{n}$. By their construction, the functions $h^{p}(x)$ are monotonic increasing and bounded for $x \in E$ (and hence, continuous almost everywhere).

Let $p$ be fixed; consider an infinite sequence $\left\{s_{p \nu}\right\}$ : this sequence determines simultaneously unique numbers $x_{p}$ and $h^{p}\left(x_{p}\right)$. It follows that the correspondence

$$
x_{p} \leftrightarrow h^{p}\left(x_{p}\right)
$$

is one-to-one for each admitted $p$.

Let us write

$$
\sum_{1 \leq p \leq n} h^{p}\left(x_{p}\right)=n^{1-n} \sum_{1 \leq \nu \leq \infty} t_{\nu} \cdot n^{-n \nu}
$$

where

$$
t_{\nu}=\sum_{1 \leq p \leq n} s_{p \nu} \cdot n^{n-p}
$$

We first show that the normalizing restriction imposed on the $s_{p}$, carries with it the analogous restriction for the $t_{n}$, proving thereby 
that the right side of (6) is a unique representation of real numbers to the base $n^{n}$. That is, we demonstrate that $t_{\nu}<n^{n}-1$ for infinitely many $\nu$, unless $t_{\nu}=n^{n}-1$ for all $\nu$.

The specified domain of the $s_{p \nu}$ is $0 \leqq s_{p \nu} \leqq n-1$; accordingly we have

$$
0 \leqq \sum_{1 \leqq p \leqq n} s_{p \nu} \cdot n^{n-p} \leqq(n-1) \sum_{1 \leqq p \leqq n} n^{n-p}=n^{n}-1 .
$$

Since $s_{p \nu}<n-1$ for infinitely many $\nu$,

$$
t_{\nu}=\sum_{1 \leqq p \leqq n} s_{p v} \cdot n^{n-p}<n^{n}-1
$$

for infinitely many $\nu$, unless $s_{p \nu}=n-1$ for all values of $p$ and $\nu$.

To complete the proof of (i) it remains, therefore, only to show that the correspondence

$$
\left(x_{1}, \cdots, x_{n}\right) \rightarrow \sum_{1 \leq p \leqq n} h^{p}\left(x_{p}\right)
$$

is one-to-one. We demonstrate, namely, that the right side of (6) determines a unique point in $E^{n}$ :

Let

$$
\sum_{1 \leqq p \leqq n} h^{p}\left(y_{p}\right)=n^{1-n} \sum_{1 \leq \nu \leqq \infty} t_{\nu}^{\prime} \cdot n^{-n \nu}
$$

if

$$
\sum_{1 \leq p \leqq n} h^{p}\left(x_{p}\right)=\sum_{1 \leqq p \leqq n} h^{p}\left(y_{p}\right)
$$

then $t_{\nu}=t_{\nu}^{\prime}$ for all values of $\nu$.

By the definition of the summands in (10), this equation is equivalent to the statement that

$$
t_{1}-t_{1}^{\prime}=\sum_{2 \leqq v \leqq \infty}\left(t_{\nu}^{\prime}-t_{v}\right) \cdot n^{n-n v}=\alpha,
$$

where $|\alpha| \leqq 1$, as shown with a simple calculation. That this inequality is strict follows from the fact that $|\alpha|=1$ if and only if $\left|t_{\nu}^{\prime}-t_{\nu}\right|$ $=n^{n}-1$ for all $\nu \geqq 2$, and then, according to the normalizing restriction, $\left|t_{1}^{\prime}-t_{1}\right|=n^{n}-1=1$. The last equality is clearly impossible.

We now prove the assertion made, that $t_{\nu}=t_{\nu}^{\prime}$ for all $\nu$, by induction on $\nu$. Since $|\alpha|<1$ and the difference $\left|t_{1}^{\prime}-t_{1}\right|$ is integral or zero, 
it follows that $\alpha=0$ and, hence, $t_{1}=t_{1}^{\prime}$. Suppose now that $t_{\nu}=t_{\nu}^{\prime}$ for all $\nu \leqq k$, where $k \geqq 1$. Then equation (10) is equivalent to the statement that

$$
t_{k+1}-t_{k+1}^{\prime}=\sum_{k+2 \leq \nu \leq \infty}\left(t_{\nu}^{\prime}-t_{\nu}\right) \cdot n^{n(k+1-\nu)}=\alpha^{\prime},
$$

where $\left|\alpha^{\prime}\right| \leqq 1$. The above reasoning shows that inevitably $t_{k+1}=t_{k+1}^{\prime}$, thereby completing the induction.

Now let

$$
t_{\nu}^{\prime}=\sum_{1 \leq p \leqq n} s_{p \nu}^{\prime} \cdot n^{n-p}
$$

the relation $t_{\nu}=t_{\nu}^{\prime}$ permits us to write

$$
0 \leqq s_{n \nu}=s_{n \nu}^{\prime}+\left[n \cdot \sum_{1 \leqq p \leqq n-1}\left(s_{p \nu}^{\prime}-s_{p \nu}\right) \cdot n^{n-p-1}\right] \leqq n-1 .
$$

Since $s_{n \nu}^{\prime}$ is non-negative, and the expression in brackets is an integral multiple of $n$, this is impossible, unless $s_{p \nu}=s_{p \nu}^{\prime}$ for all admitted values of $p$. This shows that $t_{\nu} \neq t_{\nu}^{\prime}$ unless $s_{p \nu}=s_{p \nu}^{\prime}$ for all $p$, and hence the correspondence (9) is one-to-one.

The correspondence (9) maps the unit cube, $E^{n}$, onto the closed interval $\left[0, n^{1-n}\right]$, in a one-to-one manner. To each point

$$
y=\sum_{i \leqq p \leqq n} h^{p}\left(x_{p}\right)
$$

in this interval, the assignment $g(y)=f\left(x_{1}, \cdots, x_{n}\right)$, therefore, is defined uniquely. The function $g$ is that demanded in part (ii) of our theorem.

\section{BIBLIOGRAPHY}

1. V. I. Arnol'd, On the representability of a function of two variables in the form $\chi[\phi(x)+\psi(y)]$, Uspehi Mat. Nauk. 12 (1957), no. 2(74), 119-121. (Russian)

SYRACUSE UNIVERSITY 